\title{
émulations
}

\section{Manuel Cervera-Marzal - Les nouveaux désobéissants : citoyens ou hors-la-loi ?}

Elisabeth Lagasse de Locht

Émulations - Revue de sciences sociales

2016, « Comptes rendus critiques, En ligne»

\section{Article disponible à l'adresse suivante}

https://ojs.uclouvain.be/index.php/emulations/article/view/7233

\section{Pour citer cet article}

Elisabeth Lagasse de Locht, " Manuel Cervera-Marzal — Les nouveaux désobéissants: citoyens ou hors-la-loi ? », Émulations, en ligne. Mise en ligne le 19 septembre 2016. DOI : 10.14428/emulations.cr.025

Distribution électronique : Université catholique de Louvain (Belgique) : ojs.uclouvain.be

(C) Cet article est mis à disposition selon les termes de la Licence Creative Commons Attribution, Pas d'Utilisation Commerciale 4.0 International. http://creativecommons.org/licenses/by-nc/4.0/

Éditeur : Émulations - Revue de sciences sociales / Presses universitaires de Louvain https://ojs.uclouvain.be/index.php/emulations

ISSN électronique : 1784-5734

UCL PRESSES 


\title{
Manuel Cervera-Marzal - Les nouveaux désobéissants : citoyens ou hors-la-loi?
}

\author{
Elisabeth Lagasse de Locht $^{1}$
}

Recensé : Manuel Cervera-Marzal, Les nouveaux désobéissants : citoyens ou hors-laloi ?, Paris, Le Bord de l'eau, (« La Bibliothèque du MAUSS »), 2016, 160 p.

Quelle est la légitimité de la désobéissance civile en démocratie, et quelle doit être sa place ? C'est à ces questions que tente de répondre l'ouvrage de Manuel Cervera-Marzal. L'auteur, jeune docteur en science politique, publie ici une partie de sa thèse, soutenue en 2014 à l'Université libre de Bruxelles et à l'Université Paris-Diderot, portant sur la philosophie de la désobéissance civile sous l'angle de la théorie politique. Il a notamment publié Miguel Abensour, critique de la domination, pensée de l'émancipation (2013), Gandhi. Politique de la non-violence (2015) et Pour un suicide des intellectuels (2016). Articulant la philosophie, la science politique et la sociologie, il mobilise dans sa réflexion des auteurs tels que Thoreau, Zinn, Arendt, Gandhi, Castoriadis. Il ne s'appuie cependant pas sur des travaux de sociologie plus empiriques qui portent sur cette question, tel que l'ouvrage récent d'Ogien et Laugier (2010).

Partant du constat qu'on assisterait à une « extra-légalisation des luttes d'émancipation » (p. 23), c'est-à-dire que tout un répertoire d'actions collectives serait de plus en plus marqué par l'illégalité, la thèse de l'auteur consiste à affirmer que les «nouveaux désobéissants » sont des citoyens à part entière, voire qu'ils renouvellent la signification de la citoyenneté. Pour mener à bien cette réflexion, l'auteur s'inscrit d'emblée dans une perspective de pensée de l'émancipation qui considère que «la liberté n'est pas un acquis des révolutions passées, mais une tâche à laquelle nous sommes présentement confrontés » (p. 7). Dès lors, plutôt qu'un livre sur la désobéissance civile en soi, il s'agit davantage d'une réflexion de théorie critique sur la démocratie à partir de la désobéissance citoyenne aux lois, éclairant notamment la question de son rapport avec ses institutions et la place que peut, et doit, y trouver le conflit. En effet, selon l'auteur la conflictualité sociale n’a pas diminué, mais est reconfigurée par l'action de ces désobéissants qui introduisent un nouveau rapport aux lois. Cette réflexion contribue ainsi à l'élaboration d'une philosophie de la non-violence qui se distingue du pacifisme en plaçant la lutte contre les inégalités et pour la démocratie au centre de celle-ci.

${ }^{1}$ Doctorante au Centre de recherches interdisciplinaires Démocratie, Institutions et Subjectivité (CriDIS) et au sein du groupe de recherche « Social Movements à l'Age Global » (SMAG) à I'Université catholique de Louvain. 
La démonstration de la thèse selon laquelle la désobéissance civile est un acte de citoyenneté se fait en trois temps. La première partie de l'ouvrage renverse la question de la justification de la désobéissance civile en démocratie, " pourquoi désobéir ? ", en «pour quoi désobéir ?». L'auteur commence ainsi par déconstruire l'idée que nos régimes occidentaux sont des démocraties. D'une part, il affirme que nos États sont en réalité des oligarchies, «au sens où ils se caractérisent par la domination d'une minorité sur la majorité » (p. 44). De cette façon, la présomption de culpabilité attachée aux désobéissants se déplace pour charger les États, et oppose leur violence (qui se traduit notamment dans le principe de délégation et par les inégalités sociales) à la non-violence de la désobéissance. D’autre part, il considère la démocratie comme un processus inachevé, un mouvement de démocratisation en transformation perpétuelle.

Ces bases posées, l'auteur poursuit sa réflexion sur ce qu'est la démocratie, invitant à la repenser à partir de la désobéissance civile, qu'il aborde dans la deuxième partie de l'ouvrage. Cervera-Marzal commence par y affirmer une nécessaire désacralisation des lois. Plutôt que de constituer un socle solide sur lequel s'appuyer, elles doivent être envisagées comme émanant de rapports de force qu'il est possible de faire évoluer. Il appelle donc à faire passer le combat pour l'autonomie avant celui pour la justice. La désobéissance civile est en ce sens un « acte d'auto-institution démocratique par lequel la collectivité se soulève contre la tendance d'une loi à s'autonomiser des activités sociales dont elle procède» (p. 79). Pour autant, il ne s'agit pas de se passer de lois. Au contraire, cette tension entre ordre et désobéissance, instituant et institué, est constitutif de la démocratie qui se doit de maintenir le conflit ouvert. L'auteur insiste : « il est toujours possible de désobéir, mais il n’est pas possible de toujours désobéir » (p. 81).

Si le conflit doit toujours être ouvert, quel rapport entretient-il avec la violence ? Dans la troisième et dernière partie, Cervera-Marzal refuse tout autant une vision belliciste de la démocratie, qui cherche à éliminer l'opposant, qu'une vision pacifiste, qui évacue le conflit. Il nomme "politique de la civilité » un rapport au conflit qui s'entretient dans une relation d' « adverse amicalité » (p. 106), où l'adversaire ne l'est pas du fait de sa personne, mais de par les actes qu'il pose. L'auteur s'oppose ici explicitement à la fois à une "vision ordonnée et ordonnatrice de la démocratie », dans laquelle le conflit est un « symptôme de disfonctionnement », mais aussi à la conception de la philosophie libérale (représentée par Rawls ou Habermas notamment) qui reconnaît l'existence d'un conflit, mais considère que la politique vise à réconcilier les parties. Face au pacifisme, la reconnaissance du conflit comme facteur de liberté est mise en avant ; la politique devient alors le rapport entre les luttes. Dès lors, l'auteur conclut que les nouveaux désobéissants, en agissant de façon non-violente au nom d'un idéal de justice et liberté de toutes et tous, constitueraient une figure actuelle exemplaire d'une citoyenneté renouvelée, qui ne se caractérise plus par un rapport aux frontières, mais par le fait de faire passer cet idéal devant sa propre vie.

Si cette thèse est défendue de façon assez claire et convaincante tout au long de l'ouvrage, ce dernier reste avant tout très théorique et trop peu ancré dans la réalité qu'il 
souhaite expliquer. Sans prêter à l'auteur des intentions qui ne sont pas les siennes dans cet ouvrage, le lecteur peut regretter la faible place laissée à l'enquête et aux données empiriques dans cette réflexion. En effet, au terme de cet ouvrage, la figure du nouveau désobéissant apparaît comme étonnamment pure et unifiée. Or, les tensions et débats présents sur le terrain et parmi les acteurs sont particulièrement riches pour comprendre les enjeux concrets du changement social. Ainsi, si l'auteur définit la désobéissance civile de façon relativement tranchée, notamment par la non-violence, cette question n'est jamais totalement évacuée sur le terrain, toujours en débat. Il pourrait être également intéressant de détailler davantage les différentes conceptions des acteurs qui choisissent ce mode d'action, comme d'autres ont pu le faire, par exemple en s'intéressant aux acteurs de l'économie solidaire (Frère, 2009) ou à ceux du mouvement altermondialiste (Pleyers, 2010). Cette question n'est pas sans lien avec celle de la stratégie et de la place de la désobéissance civile dans le changement social, qui semble trop rapidement laissée de côté par l'auteur. Celle-ci nous apparaît d'ailleurs liée de façon déterminante à la problématique de l'accès à ce mode d'action qui, par les risques qu'il engendre, exclut potentiellement des acteurs plus vulnérables. Dès lors, si ce livre a le mérite d'éclairer efficacement et de façon accessible les débats théoriques que soulève la désobéissance civile, notamment sur les questions de la citoyenneté, de la violence et de leurs rapports, il nous semble qu'un vide reste à combler, notamment par des recherches se focalisant davantage sur les acteurs de la désobéissance, afin d'enrichir la description et la compréhension des enjeux et des pratiques liées au changement social, en cherchant à plus concrètement « enfiler les lunettes » des acteurs (p. 52).

\section{Bibliographie}

Cervera-Marzal, M. (2006), Miguel Abensour, critique de la domination, pensée de l'émancipation, Paris, Sens et Tonka.

Cervera-Marzal, M. (2015), Gandhi. Politique de la non-violence, Paris, Michalon.

Cervera-Marzal, M. (2016), Pour un suicide des intellectuels, Paris, Textuel.

FRÈRE, B. (2009), Le nouvel esprit solidaire, Paris, Desclée de Brouwer.

Ogien, A., Laugier, S. (2010), Pourquoi désobéir en démocratie ?, Paris, La Découverte.

Pleyers, G. (2010), Alter-Globalization. Becoming Actor in the Global Age, Cambridge, Polity Press. 